\title{
Intrachromosomal Amplification of Chromosome 21
}

National Cancer Institute

\section{Source}

National Cancer Institute. Intrachromosomal Amplification of Chromosome 21. NCI

Thesaurus. Code C124874.

A cytogenetic abnormality that refers to the allelic gain of an internal part of chromosome 21. It is a rare high-risk chromosomal abnormality that occurs in approximately $2-5 \%$ of pediatric patients with B-cell precursor Acute Lymphoblastic Leukemia. This abnormality has been associated with a poor outcome in patients treated by standard protocols. 